REVIEW

\title{
The epidemiology of tuberculosis in Europe
}

\section{Tony Walls, Delane Shingadia}

Arch Dis Child 2007;92:726-729. doi: 10.1136/adc.2006.102889

$\mathrm{T}$ uberculosis (TB) continues to be one of the most devastating and widespread infections in the world. It is estimated that in 2004 there were 8.9 million new cases of TB globally. ${ }^{1}$ In general, children make up only a small proportion of cases, but in some high-incidence countries up to $15 \%$ of all TB cases occur in children. ${ }^{2}$ In June 2005 the World Health Organization (WHO) approved a new Stop TB Strategy with the aim of dramatically reducing the global burden of TB by $2015 .^{3}$ Their intention is to cure at least $85 \%$ of sputum smear-positive cases and reduce the prevalence of and deaths due to TB by $50 \%$ relative to the rates in 1990 .

The WHO Global tuberculosis control report shows that the greatest burden of TB is in sub-Saharan Africa and Asia, with TB case notifications in the WHO European Region constituting less than 10\% of worldwide notifications. ${ }^{1}$ While notifications are an excellent source of information on adult disease, an accurate description of the burden of $\mathrm{TB}$ in children is very difficult to obtain. The WHO notification criteria include only cases that are sputum smear positive. Fewer than 15\% of children with culture-proven $\mathrm{TB}$ will be sputum smear positive, with the result that only a small percentage of children with TB will be represented in the data. The quality of information provided to the WHO can also vary and in many countries surveillance data are often unreliable due to poor diagnostic facilities and reporting systems. Because of these issues the International Union Against Tuberculosis and Lung Disease has stated that reliable information on the incidence of TB in childhood can only be obtained in developed countries, which have better diagnostic and reporting systems. ${ }^{4}$

In Europe there is a huge disparity in the rates of TB between the western and eastern countries, and it appears to be worsening. ${ }^{6}$ Even in countries with low TB prevalence, rates of disease differ between urban and non-urban settings. Young children are the most likely to develop disease after infection and are significantly more likely to develop extrapulmonary and severe disseminated disease than adults. ${ }^{5}$ Children also develop disease sooner after infection than adults and thus the occurrence of disease in children provides an early indication of transmission of TB within communities.

$\mathrm{TB}$ surveillance in Europe is co-ordinated by EuroTB, which is a network of national institutions in charge of TB surveillance in the 53 countries of the WHO European Region. ${ }^{6}$ The organisation aims to improve the public health surveillance and control of $\mathrm{TB}$ in Europe by promoting standardisation of surveillance methods and annual data collection. It collects information on TB case surveillance, drug resistance and treatment outcomes. The case definitions used are similar to those used by the WHO but also include criteria for reporting other-than-definite TB cases (formally classified as probable cases). This allows children, many of whom may have a clinical diagnosis of TB without microbiological confirmation, to be included in the data. This review will focus on the epidemiology of paediatric $\mathrm{TB}$ in Europe, using data largely from EuroTB but also from other sources.

\section{TB NOTIFICATIONS IN THE WHO EUROPEAN REGION}

Overall, the rates of TB in Europe for both adults and children have been increasing steadily in the last decade. ${ }^{6}$ Notification rates for the entire region have risen from 74.4 cases/100 000 population in 1997 to $104 / 100000$ population in 2004. The WHO European Region is divided into three geographic regions by EuroTB. ${ }^{6}$ The Western European region includes countries in the European Union, as well as seven other closely related countries (Andorra, Iceland, Israel, Monaco, Norway, San Marino and Switzerland). Eastern Europe is predominantly made up of countries of the former Soviet Union, with a large proportion of the population from the Russian Federation. Central Europe incorporates the eight countries in between these two regions (Albania, Bosnia and Herzegovina, Bulgaria, Croatia, Macedonia, Romania, Serbia and Montenegro, and Turkey).

The overall EuroTB case notifications reveal alarming disparities in the rates of TB between countries in Western Europe and those in Eastern Europe, comprising mostly states of the former Soviet Union (table 1). ${ }^{6}$ For example, figures for 2004 show both Ireland and the UK had notification rates for all cases of $<13 / 100000$ population. This is compared to Romania and the Russian Federation whose rates are $>100 / 100000$ and Kazakhstan with a rate of 216/100 000. Case notification rates in Ireland and the UK have fallen since 1980 and in the last 5 years have remained relatively unchanged. In contrast, many Eastern European countries have had steadily increasing rates of TB notifications. In 1995, the Russian Federation had 84980 notifications, increasing to 152438 by 2004. In the same period, notifications in Kazakhstan rose from 11310 to 32 131. TB notifications in the countries of Central Europe fall somewhere in between these two extremes and while many of these countries have falling rates of $\mathrm{TB}$, increases of $2-4 \%$ annually since 1995 have occurred in Bulgaria and Bosnia and Herzegovina.

Abbreviations: $\mathbb{I N H}$, isoniazid; $M D R$, multi-drug resistance; TB, tuberculosis; WHO, World Health Organization 


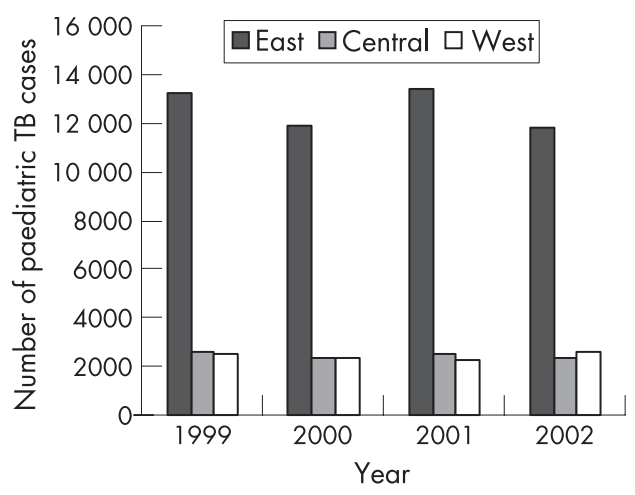

Figure 1 Number of paediatric TB cases in the WHO European Region, 1999-2002 (EuroTB).

\section{PAEDIATRIC TB IN EASTERN EUROPE}

Rates of TB in children are very high in Eastern Europe, although age-specific rates are not available for all countries. Between 1999 and 2002, almost two thirds of TB notifications in Europe for children aged 0-14 years occurred in the Eastern European region (fig 1) with 12-13 000 cases per year reported (2003-4 is not included as age-specific data from the Russia Federation were unavailable). This is despite this region having less than one third of the total WHO European Region population. In 2004, Kazakhstan reported more TB cases than the entire European Union (EU) for children aged 5-14 years.

Paediatric TB is generally a direct result of transmission from a smear-positive adult, and higher rates of paediatric TB might be expected in high-burden countries where there are high rates of TB transmission within communities. However, it is not just the prevalence of TB in the communities but the age distribution of adult $\mathrm{TB}$ in these countries which contributes to the high rates in children. In many Eastern European countries, the highest rates of adult disease occur in younger adults in the 25-44-year-old age bracket. ${ }^{7}$ This is in comparison to countries in Western Europe and the EU, where the highest rates of disease occur in older adults (>65 years), probably due to reactivation following infection in childhood (fig 2). A similar pattern is seen within the UK, where the highest rates of TB among adults born outside the UK occur in the 25-44-year-old age group, and in the older age groups for those born in the UK. ${ }^{8}$ The fact that more young adults in Eastern Europe have active TB is likely to mean that more children are exposed to TB from their parents or carers, and hence higher rates of disease can be expected in children. ${ }^{7}$ The explanations for the high rates of TB in Eastern Europe are complex and vary between countries. Shilova and Dye investigated the reasons for the resurgence of TB in Russia during the 1990s. ${ }^{9}$ They found that overall there had been an increasing incidence of disease, as shown by the increase in cases reported, yet there was also evidence that the detection of TB was declining. Treatment success rates had decreased over the decade, while case fatality rates for TB had significantly increased. The rates of disease in children had dropped more rapidly (6.1\% per year) than in the rest of the population $(3.7 \%$ per year) up to the beginning of the 1990s. However, since then increasing rates in the overall population have been mirrored in the higher rates of TB in children, which have increased by approximately $9.0 \%$ per year. The authors attributed these increases to a combination of deteriorating TB control services and changes in social and economic conditions that have had an overall negative effect on Russian health in general.

\section{TB IN LOW-PREVALENCE COUNTRIES}

In most of the low-prevalence countries in Europe, such as the UK, France and Germany, overall notification rates for TB have been declining over the last 20 years. ${ }^{1}$ National survey data from the UK show a decrease in TB rates in all age groups from 1978-9 onwards, reaching their lowest levels in the mid-1980s.

National rates in children and young people overall have remained relatively low but have been gradually increasing in London in the last 5 years, along with overall TB incidence. ${ }^{10}$ However, despite small increases nationally, there have been specific changes in the patterns and distribution of paediatric

Table 1 TB incidence and notification rates, and drug resistance in selected countries from the EU Western Europe, Central Europe and Eastern Europe in 2004

\begin{tabular}{|c|c|c|c|c|c|}
\hline Country & $\begin{array}{l}\text { Number } \\
\text { of cases }\end{array}$ & $\begin{array}{l}\text { Number of patients } \\
<15 \text { years old } \\
\text { (\% of total) }\end{array}$ & $\begin{array}{l}\text { Rate per } \\
100000 \\
\text { population }\end{array}$ & $\begin{array}{l}\% \text { Isoniazid } \\
\text { resistant }\end{array}$ & $\begin{array}{l}\% \text { Multi-drug } \\
\text { resistant }\end{array}$ \\
\hline \multicolumn{6}{|l|}{ EU and the West } \\
\hline United Kingdom & 7584 & $441(5.8)$ & 12.7 & 5.2 & 0.8 \\
\hline Czech Republic & 1057 & $7(6.6)$ & 10.3 & $1.4^{*}$ & $0.5^{\star}$ \\
\hline Denmark & 385 & $28(7.2)$ & 7.1 & 6.1 & 0.0 \\
\hline Estonia & 594 & $2(3.4)$ & 44.5 & 29.4 & 20.4 \\
\hline Germany & 6583 & $269(4.1)$ & 8.0 & 3.9 & 0.4 \\
\hline Ireland & 437 & $9(2)$ & 10.7 & $0.9^{*}$ & $0.0^{*}$ \\
\hline Italy & 4220 & $197(4.6)$ & 7.3 & $10.0^{*}$ & $2.7^{*}$ \\
\hline Latvia & 1610 & $110(6.8)$ & 69.4 & 31 & 17.7 \\
\hline Lithuania & 2514 & 119 (4.7) & 73 & 31.9 & 19.6 \\
\hline Spain & 6392 & $356(5.6)$ & 15 & $8.4^{*}$ & $3.2^{*}$ \\
\hline Portugal & 3873 & $76(2)$ & 37.1 & $8.0^{*}$ & $1.7^{*}$ \\
\hline \multicolumn{6}{|l|}{ Central Europe } \\
\hline Bosnia and Herzegovina & 2382 & 37 (1.5) & 60.9 & 1.6 & 0.9 \\
\hline Croatia & 1297 & $44(3.4)$ & 28.6 & 1.0 & 0.4 \\
\hline Romania & 31814 & $1443(4.5)$ & 146 & 14.3 & 5.3 \\
\hline Turkey & 19799 & No data & 27.4 & No data & No data \\
\hline \multicolumn{6}{|l|}{ Eastern Europe } \\
\hline Armenia & 1701 & $74(4.4)$ & 56.2 & $46.5^{*}$ & $24.0^{*}$ \\
\hline Georgia & 5967 & $355(6)$ & 132.1 & No data & No data \\
\hline Kazakhstan & 32131 & $1828(5.7)$ & 216.5 & $48.4^{*}$ & $28.0^{*}$ \\
\hline Kyrgystan & 6641 & $867(13)$ & 127.6 & $48.1^{*}$ & $27.5^{*}$ \\
\hline Russian Federation & 152438 & No data & 105.9 & No data & No data \\
\hline Turkmenistan & 4172 & $263(6.3)$ & 87.5 & No data & No data \\
\hline Ukraine & 38403 & $674(1.8)$ & 81.7 & No data & No data \\
\hline
\end{tabular}

*Culture or sensitivity testing not routinely performed. Incomplete testing nationally.

Data reproduced with the permission of EuroTB. 

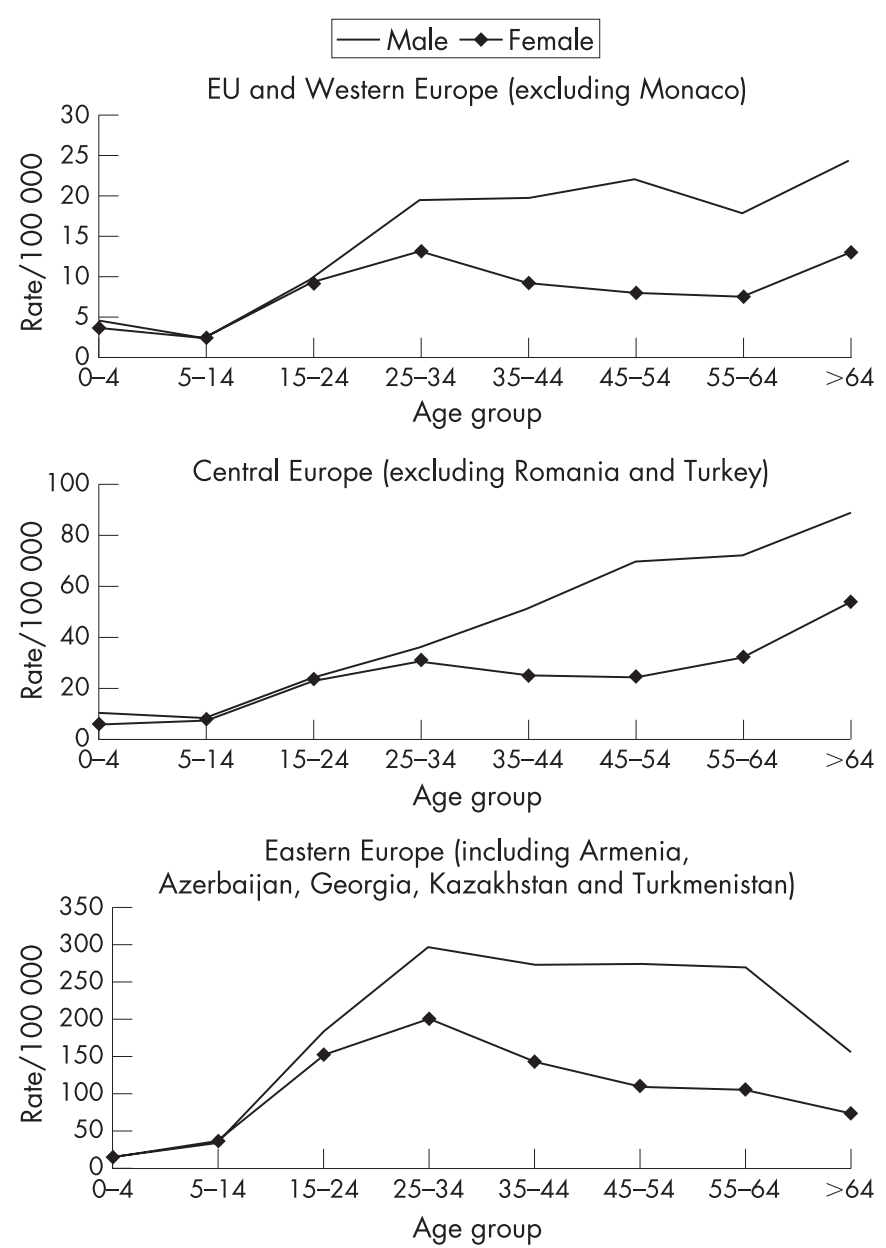

Figure 2 TB notification rates by age group and sex, 2004 (used with permission from EuroTB).

$\mathrm{TB}$ in the UK that are not reflected in national data. Overall, TB notifications have increased substantially in London, which accounts for $43 \%$ of national cases (2003 data), with a notification rate of 46/100 $000 .{ }^{8}$ Several boroughs in London now have TB notification rates $>60 / 100000$, with some having rates $>120 / 100000 .{ }^{10}$ Cases in children under 16 years of age in London have risen almost every year since 1988 with increases across all age groups. Some areas have recorded rates of $>40$ / 100000 in children. The proportion of black African children with TB in 1998 (44\%) had increased substantially from 1993 $(23 \%)$, whereas the proportion of paediatric cases from the Indian sub-continent had fallen (from $50 \%$ in 1993 to $21 \%$ in 1998). ${ }^{11}$ The change in proportions is largely related to the increase in black African children, with $95 \%$ of TB cases in this group resident in London. ${ }^{12}$ In 2004, the British Paediatric Surveillance Unit Childhood Tuberculosis Study found the TB notification rate for black African children (74.7/100 000) was by far the highest in the UK (white children 0.87/100 000, Indian sub-continent children 20.2/100 000). ${ }^{13}$ Similar patterns have occurred in other low-prevalence countries in Europe. Eriksson et al reviewed the epidemiology of paediatric TB in Stockholm between 1976 and 1995. ${ }^{14}$ They found the overall rates of TB in children increased from $<1 / 100000$ early in the study to 5.8/100 000 between 1991 and 1995. The increase in case notifications was mostly due to children born outside the country ( $50 \%$ of children with TB were from Africa) or who had at least one parent who was born abroad. There were no cases of TB in children whose parents were both Swedish born in the years 1991-5. A similar study in Copenhagen between 1984 and 1993 found that 70\% of children with TB had immigrant parents. ${ }^{15}$ This is in contrast to Eastern Europe where nearly all childhood TB occurs in children born locally. ${ }^{6}$

\section{SITE OF TB DISEASE IN CHILDREN}

EuroTB data from 2004 suggest there have been some changes in the site of TB disease for children in the WHO European Region in recent years. ${ }^{6}$ The proportion of children with pulmonary disease in 2004 was $55.3 \%$ compared to $68.1 \%$ in 2000. In comparison, rates for adults have remained unchanged during this time, with approximately $80 \%$ of cases involving pulmonary disease. The increasing proportion of extra-pulmonary TB in children is mostly due to the identification of intrathoracic lymph disease (classified as extra-pulmonary TB for surveillance). In 2004, $25 \%$ of childhood cases had intrathoracic lymph node disease compared to the overall rate of $2.4 \%$. This is likely to be due to higher rates of primary disease in children, manifesting as intra-thoracic lymphadenopathy, compared to reactivation of latent disease, which is more common in adults. It is unclear whether the increasing proportion of extra-pulmonary disease in children is due to increasing rates of primary infection, or simply the result of increased identification of intra-thoracic lymph node disease.

The other notable difference between rates of extra-pulmonary disease in adults and children is for TB meningitis. In 2004, TB meningitis made up $1.7 \%$ of all disease in children aged $0-$ 14 years compared to $0.6 \%$ of TB in adults. ${ }^{6}$

\section{TB AND MIGRATION}

During the latter half of last century there was an unprecedented movement of people between countries. Molecular epidemiological studies undertaken in Norway and the UK both suggest that many of the new TB cases in immigrants are due to reactivation of infections acquired abroad. ${ }^{16}{ }^{17}$ In the UK, 61\% of $\mathrm{TB}$ cases reported in 2004 occurred in foreign-born residents. In the UK, the greatest proportion of immigrants with TB have historically been from African countries and the Indian subcontinent. However, new EU states such as Estonia and Latvia have some of the highest rates of TB in Europe. In Israel during the mid-1990s, recent immigrants from Russia made up a substantial proportion of new TB cases. They accounted for over half of new cases in southern Israel and had substantially higher rates of drug-resistant disease. ${ }^{18}$ Prior to the EU expansion in 2004, the reported TB incidence in the 10 soonto-be new EU countries had been approximately double that of existing EU countries. ${ }^{19}$ Increasing immigration and movement to and from these countries within Europe could lead to higher rates of imported TB from these states.

Immigrant children born in countries with high rates of TB are at much higher risk of developing TB for several reasons. Firstly, those who are recent arrivals may have been exposed to TB in their home country. The number of new cases of TB is much higher in children within 5 years of arrival in the UK when compared to those who have been in the country longer (authors' BPSU data, manuscript in preparation). A similar pattern was seen in Israel, where a recent study found that $80 \%$ of TB in immigrant children was diagnosed within 3 years of arrival. ${ }^{20}$

Secondly, the most likely source of TB in children is from a family contact, and future TB exposure from a smear-positive family member is more likely in these children. The long time between infection and reactivation of TB in adults means that the increased risk of disease in children extends to those whose parents were born overseas even if they themselves were born in a low-risk country.

\section{HIV AND TB}

HIV infection has had a profound effect on the incidence of TB globally, particularly in sub-Saharan Africa where HIV infection rates are high. HIV is known to greatly increase the annual risk of 
progression from TB infection to active disease and TB is the commonest opportunistic infection in people with HIV infection. Increased rates of $\mathrm{TB}$ in children have been associated with increased rates of disease among HIV-infected adults in the community. ${ }^{21}$ This effect may be indirect, with these children being more likely to have close contact with a smear-positive adult than non-HIV-infected children. ${ }^{22}$ The recorded rates of TB and HIV co-infection for adults are highly variable but tend to be highest in Western Europe. In the small number of countries who routinely record HIV status in TB cases, $4.4 \%$ of patients were coinfected in Estonia, 15.7\% in Portugal, 5.1\% in Spain, and 0\% in Slovakia (2004 data). ${ }^{6}$ Few of the Eastern and Central European countries routinely report HIV status in TB patients. It seems likely that with increasing rates of HIV in the Russian Federation and high rates of $\mathrm{TB}$ that rates of co-infection would be high. However, to date there is little published evidence to support this.

At present, there is little information on how the HIV pandemic has affected the incidence of TB in children in the European region and how co-infection differs between Western, Central and Eastern European countries.

\section{DRUG-RESISTANT TB}

Drug-resistant TB is of great importance worldwide as it reflects on the control of TB in the population, and also makes therapy more expensive and more difficult to implement. When it occurs in children, it will usually be primary resistance, as children are generally less likely to have been treated previously. In most children, definite confirmation of drugresistant TB may not be possible, due to lower rates of microbiological identification from paediatric samples. Often the diagnosis of drug-resistant TB will be made on the basis of confirmation of drug resistance in the index case, usually a household contact. In high-prevalence communities, however, children may have multiple exposures within the same household, so even if close contacts have drug-sensitive disease the possibility of resistant disease in the child still exists.

At present there is little evidence to suggest that drug-resistant $\mathrm{TB}$ is more infectious or more likely to cause disease than drugsensitive TB. ${ }^{23}$ In general, the incidence and types of resistant organisms encountered in children will reflect the organisms circulating in the community. A large South African study, conducted between 1994 and 1998 in TB culture-positive children, found $5.6 \%$ had isoniazid (INH) resistance and $1 \%$ had multi-drug resistance (MDR, defined as resistance to both INH and rifampicin). ${ }^{24}$ These results were essentially the same as surveillance data from adults with TB during the same period, where $3.9 \%$ had INH resistance and $1.1 \%$ MDR. In the UK between 1993 and 1999, the rate of INH resistance in paediatric cases was $6.3 \%$, and of MDR TB was $0.8 \%$, again similar to the rates in adults of $5.7 \%$ and $1.2 \%$, respectively. ${ }^{25}$ At present there are limited data on the rates of MDR TB in children in Europe, and thus the best approximation comes from combined adult and paediatric data. Of countries who routinely carry out sensitivity testing on all TB isolates, three countries stand out as having very high rates of multi-drug resistant TB. In 2004, the rates of MDR TB were $20.4 \%, 17.7 \%$ and $19.6 \%$ for Estonia, Latvia and Lithuania, respectively. ${ }^{6}$ This compares with rates of $<1 \%$ for almost all other countries in Western Europe. Sensitivity testing is generally not complete in Eastern European countries, but in the few countries who have reported data to EuroTB, rates of MDR TB are very high. In 2004, the rates of MDR TB were $24 \%, 28 \%$ and $27.5 \%$ for Armenia, Kazakhstan and Kyrgyzstan, respectively. ${ }^{6}$

\section{SUMMARY}

Although TB in Europe makes up a small percentage of the disease globally, its epidemiology reflects worldwide trends. There are major disparities between the rates of disease between resource-poor Eastern countries and those countries in the West who have the resources to fund TB control programmes. In the countries of Western Europe, increasing rates of paediatric $\mathrm{TB}$ in some areas, particularly large metropolitan centres, can be largely attributed to transmission of infection from immigrants who acquire infection from their countries of origin. In contrast, countries in Eastern Europe are seeing a resurgence of TB in local-born individuals related to multiple factors including breakdown of TB control, multi-drug resistant TB and HIV infection. Rates of TB in children give an early indication of changes in the patterns of $\mathrm{TB}$ within communities because of the short time between infection and the development of disease. It is essential, therefore, that improvements be made across Europe in the diagnosis, prevention and monitoring of TB in this high-risk group.

\section{Authors' affiliations \\ Tony Walls, Delane Shingadia, Great Ormond Street Hospital for Children, Great Ormond Street, London, UK}

Competing interests: None.

\section{REFERENCES}

1 WHO. Global tuberculosis control - surveillance, planning and financing Geneva: World Health Organization, 2007, http://www. who.int/tb/ publications/global_report/en/index.html (accessed 18 April 2007).

2 Murray C, Styblo K, Rouillon A. Tuberculosis in developing countries: burden, intervention and cost. Bull Int Union Tuberc Lung Dis 1990;65:6-24.

3 Raviglione M, Uplekar M. WHO's new Stop TB Strategy. Lancet 2006;367:952-5.

4 Hershfield E. Tuberculosis in children: guidelines for diagnosis, prevention and management (a statement of the scientific committees of the IUATLD). Bull Int Union Tuberc Lung Dis 1991;66:61-7.

5 Miller F, Seal R, Taylor M. Tuberculosis in children. Boston: Little, Brown, 1963

6 EuroTB. Surveillance of tuberculosis in Europe. Report on tuberculosis cases notified in 2005. http://www.euroTB.org (accessed 18 April 2007).

7 Walls T, Shingadia D, Novelli V. The epidemiology of paediatric tuberculosis in Europe. Curr Paediatr 2004;14:258-62.

8 Health Protection Authority. Cases of tuberculosis rise steeply during 2005. Press release 2 November 2006. http://www.hpa.org.uk (accessed 18 April 2007).

9 Shilova V, Dye C. The resurgence of tuberculosis in Russia. Philos Trans R Soc Lond 2001;356:1069-75.

10 Antoine D, Maguire H, Story A. Epidemiology and response to the growing problem of tuberculosis in London. Euro Surveill 2006;1 1:25-8.

11 Atkinson $\mathrm{P}$, Taylor $\mathrm{H}$, Sharland $M$, et al. Resurgence of paediatric tuberculosis in London. Arch Dis Child 2002;86:264-5.

12 Balasegaram S, Watson J, Rose A, et al. A decade of change: tuberculosis in England and Wales 1988-98. Arch Dis Child 2003;88:772-7.

13 Teo S, Shingadia D. Tuberculosis (TB) in childhood. In: BPSU Annual Report 2004-2005. London: BPSU, 2005, 39. http://www. bpsu.inopsu.com laccessed 18 April 2007).

14 Eriksson M, Bennet R, Danielsson N. Clinical manifestations and epidemiology of childhood tuberculosis in Stockholm 1976-95. Scand J Infect Dis 1997;29:569-72.

15 Rosenfeldt V, Paerregaard A, Fuursted K, et al. Childhood tuberculosis in a Scandinavian metropolitan area 1984-93. Scand J Infect Dis 1998;30:53-7.

16 Dahle U, Sandven P, Heldal E, et al. Molecular epidemiology of Mycobacterium tuberculosis in Norway. J Clin Microbiol 2001;39:1802-7.

17 Maguire H, Dale J, McHugh T, et al. Molecular epidemiology of tuberculosis in London 1995-7 showing low rate of active transmission. Thorax 2002; 57:617-22

18 Gilad J, Borer A, Riesenberg K, et al. Epidemiology and ethnic distribution of multidrug-resistant tuberculosis in Southern Israel, 1992-1997. Chest 2000;1 17:738-43.

19 Falzon D, Infuso A. World TB Day 2004 and current TB perspectives in Europe: an update from EuroTB. Eurosurveillance Weekly. 2004;8: 040318, http:// www.eurosurveillance.org/ew/2004/040318.asp\#1 (accessed 18 April 2007).

20 Chemtob D, Weiler-Ravell D, Leventhal A, et al. Epidemiological characteristics of pediatric active tuberculosis among immigrants from high to low tuberculosisepidemic countries: the Israel experience. Isr Med Assoc J 2006;8:21-6.

21 Cantwell M, Binkin N. Impact of HIV on tuberculosis in sub-Saharan Africa: a regional perspective. Int $J$ Tuberc Lung Dis 1997;1:205-14.

22 Thomas $\mathbf{P}$, Bornschlegel K, Singh T, et al. Tuberculosis in human immunodeficiency virus-infected and human immunodeficiency-exposed children in New York City. Pediatr Infect Dis J 2000;19:700-6.

23 Tiexeira L, Perkins $M$, Johnson J. Infection and disease among household contacts of patients with multi-drug resistant tuberculosis. Int J Tuberc Lung Dis 2001;5:321-8.

24 Schaaf H, Gie R, Beyers N, et al. Primary drug-resistant tuberculosis in children. Int J Tuberc Lung Dis 2000;4:1149-55.

25 Diuretic T, Herbert J, Drobniewski F, et al. Antibiotic resistant tuberculosis in the United Kingdom 1993-1999. Thorax 2002;57:477-82. 